\title{
Studies of the Linearity of the ATLAS EM Barrel Calorimeter
} Electron Beam Test Results from 2002 and 2004

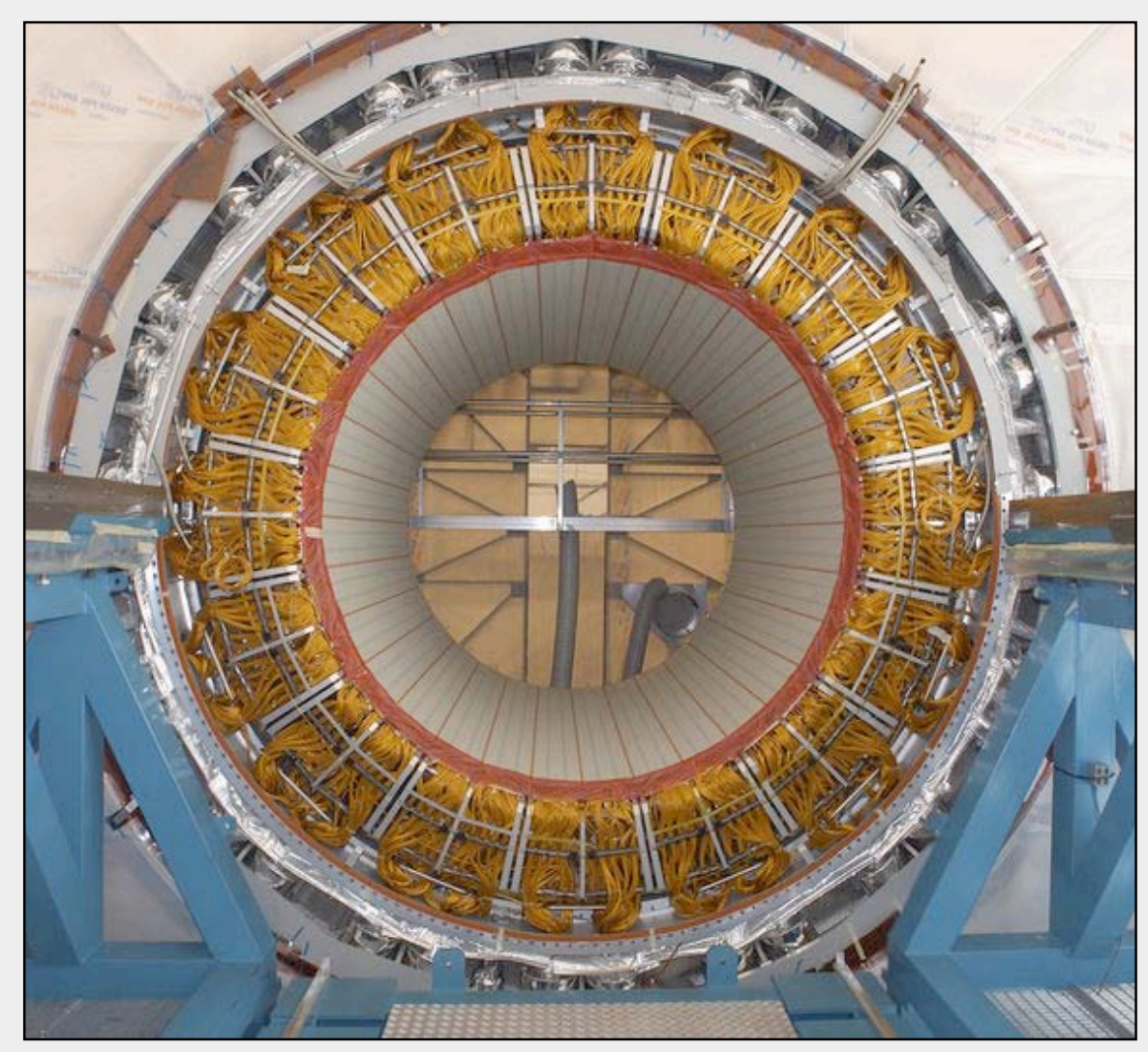

\author{
Walter Lampl \\ University of Arizona, Tucson \\ On behalf of the ATLAS \\ Liquid Argon Calorimeter \\ Collaboration
}




\section{Structure of the LAr Galorimeter}

(see talk by M. Aleksa for more details)

- Accordion Sampling Calorimeter

- Segmentation in three longitudinal compartments

- Presampler

- (Significant) amount of dead material upstream $\left(\sim 2-3 X_{0}\right)$

- Cryostat wall, solenoid, ...

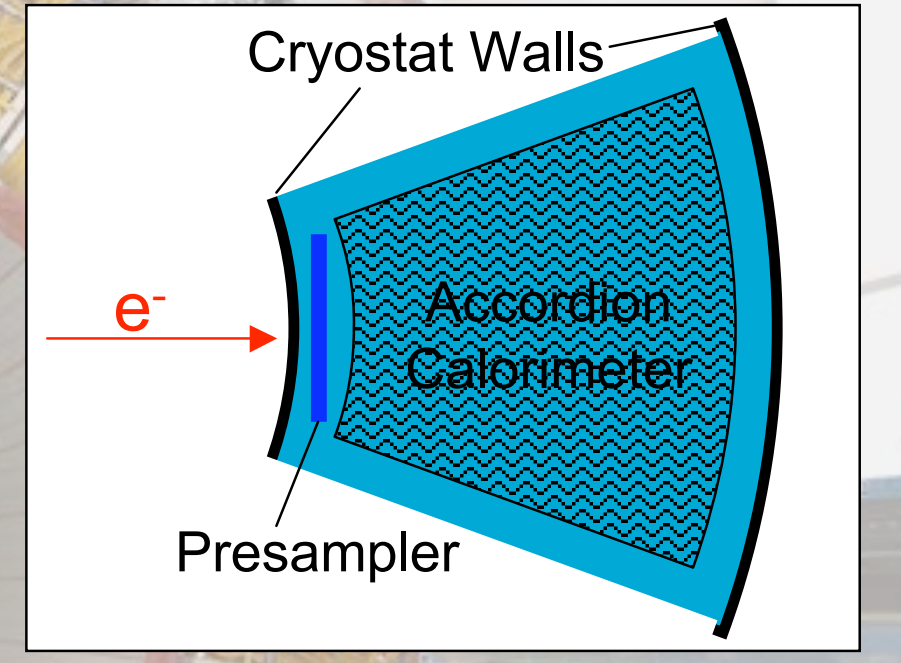

- Calibration Strategy:

- Use MC to understand effect of upstream material

- Validate MC with testbeam data

- Derive calibration constants from MC

- Cross-check by applying calibration to testbeam.
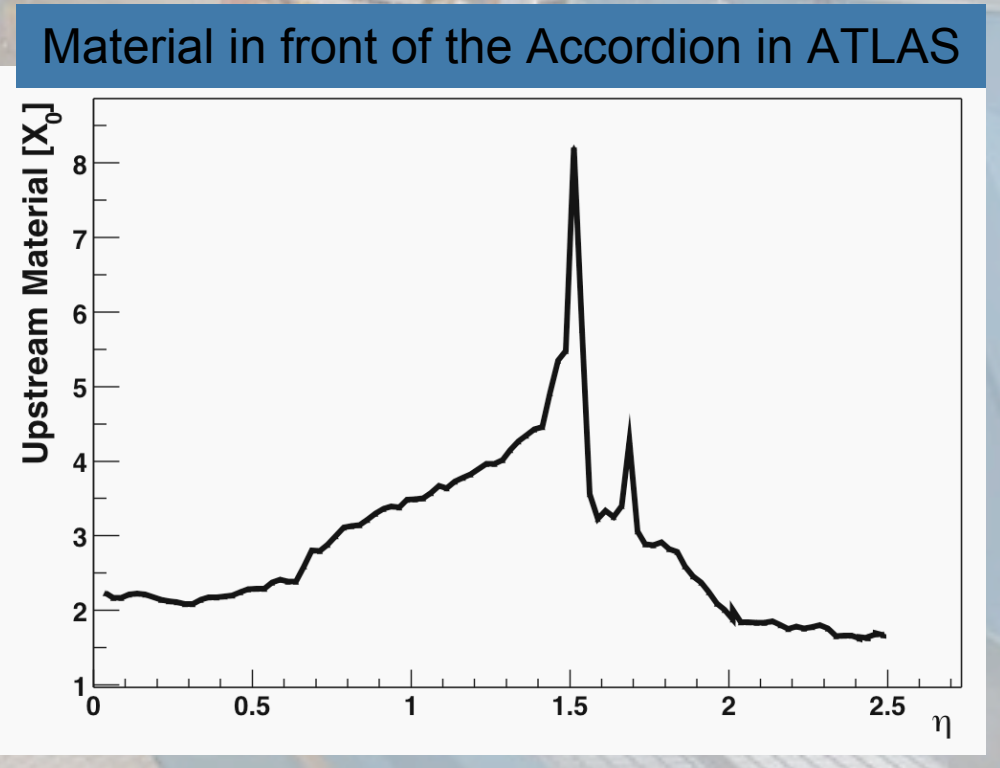


\section{Test Beam Setups}

(See talks by M. Delmastro and I. Nikolic for more details)

Electron beams from the CERN SPS H8 beam line

2002 Standalone Run

- Precision Energy Scan

- Exceptionally accurate determination of beam energy

- Dedicated beam line instrumentation

- $\sigma_{\mathrm{E}}=11 \mathrm{MeV}+3.4 \cdot 10^{-4} \mathrm{E}$

- 15 Energy-Points in the range of $10-180 \mathrm{GeV}$

- Impact point

$$
\eta=0.687, \varphi=0.282
$$

2004 Combined Run

- Energy and Material Scan

- Varied upstream material

- 2.4, 2.7, 3.0, 3.3 $\mathrm{X}_{\text {r }}$ realized by adding $25 \mathrm{~mm}$ Al plates

- 6 Energy points

$$
\text { - } 9,20,50,100,180,250 \mathrm{GeV}
$$

- Impact point

$$
\eta=0.4, \varphi=0
$$

- Very low energy

- Dedicated beam line modification

- 1 to $9 \mathrm{GeV}$

- No linearity results yet

We use a Geant4 based simulation of both setups. 


\section{Energy Deposit in the various regions}

\section{(Simulation of 2004 setup)}
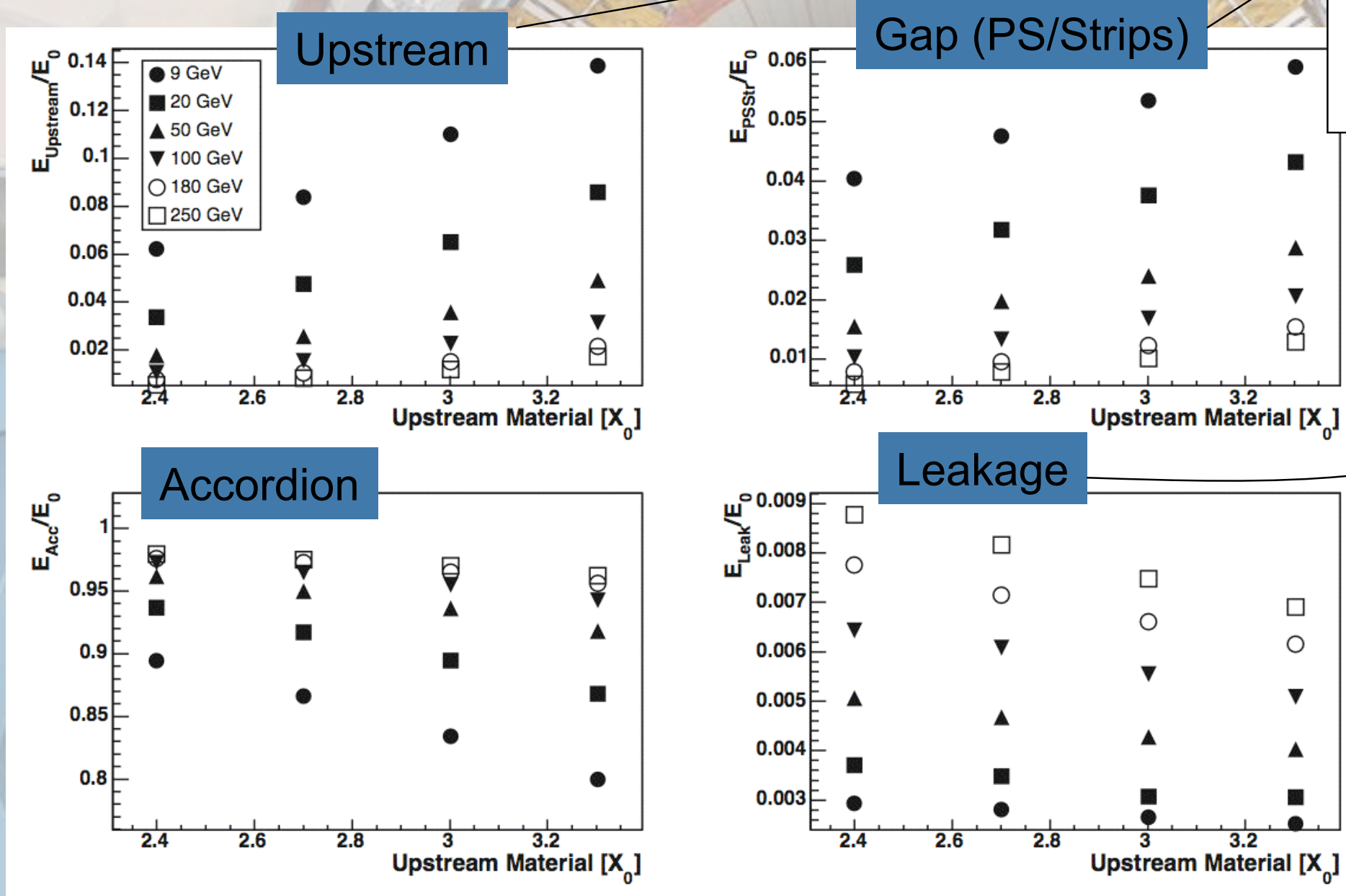

June, $7^{\text {th }}, 2006$ 


\section{Precise Calibration of the ATLAS EM Calorimeters Correcting Upstream Energy Loss}

What is the proper weight for the Presampler signal?
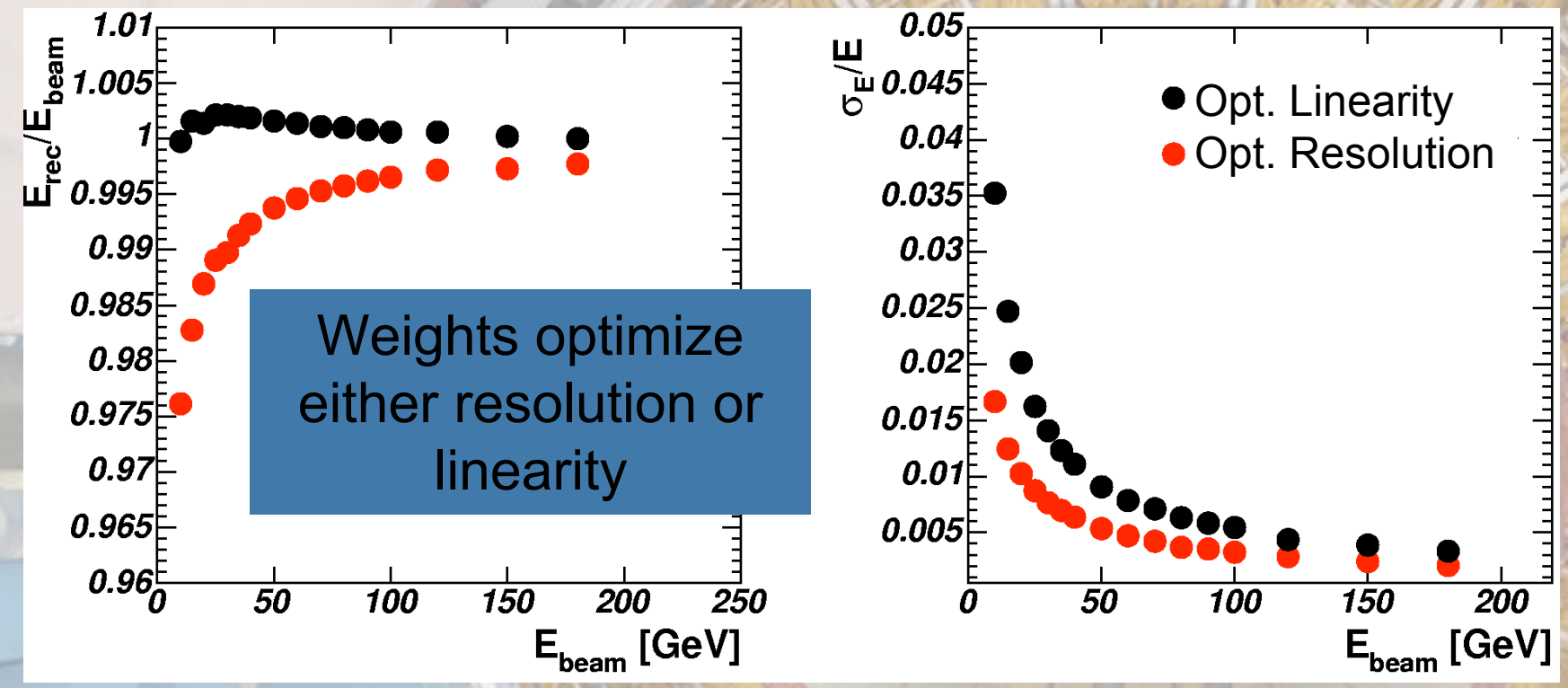

- A simple weight is not sufficient!

- Correlation plot of upstream energy deposit vs PS signal features an offset!

\section{$E_{\text {Upstream }}=a+b E_{P S}$}

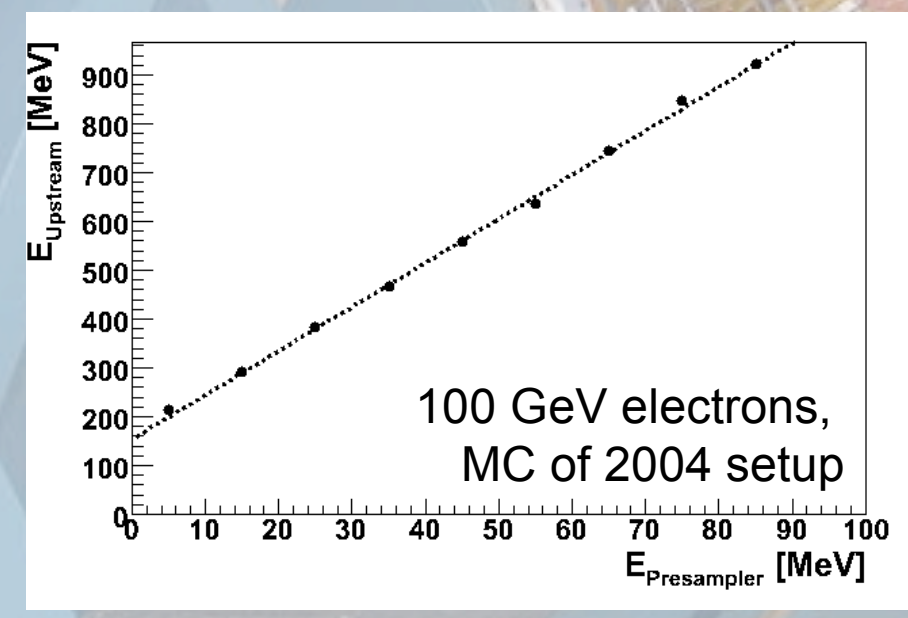

- Offset a accounts for energy loss by particles stopping before the presampler

- Ionization energy loss (roughly energy independent)

- Low-E bremsstrahlung photons that do not reach the Presampler (energy dependent)

- Photo-nuclear interactions (energy dependent)

- Weight $\mathbf{b}$ accounts for ionization energy loss by particles traversing upstream matter and (part of) the presampler. 


\section{Precise Calibration of the ATLAS EM Calorimeter Correcting for the Gap between PS and Accordion}

- Significant amount of inactive material $\left(\sim 0.5 X_{0}\right)$

- Electronics boards and cables immersed in LAr

- Dependence on impact point

- Shower already developed (about 2-3 $X_{0}$ before Accordion)

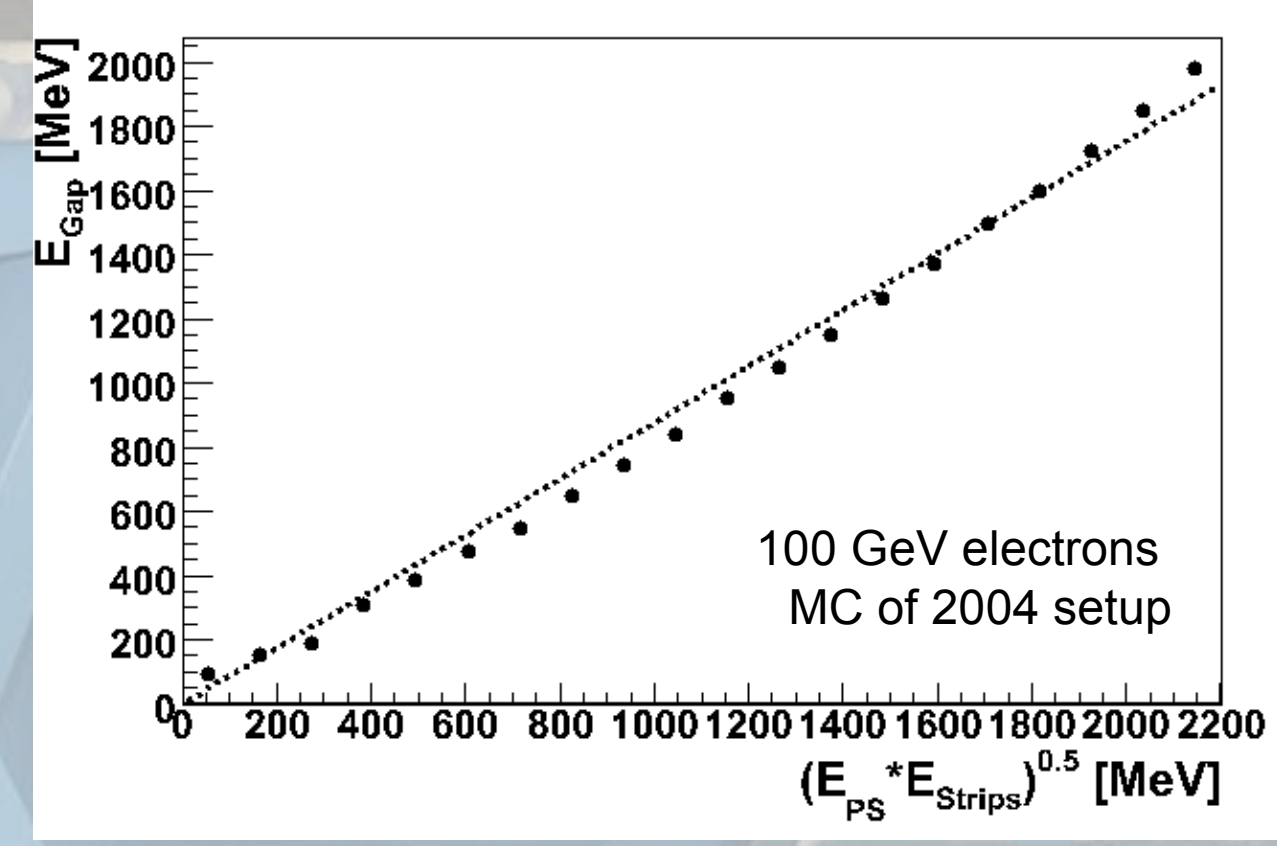

- Best correlation between measured quantities and energy deposit in the gap:

$$
E_{\text {Gap }}=c \cdot \sqrt{E_{P S} \cdot E_{1}}
$$

- Empirically found 


\section{Precise Calibration of the ATLAS EM Calorimeters}

\section{Calibrating the Accordion}

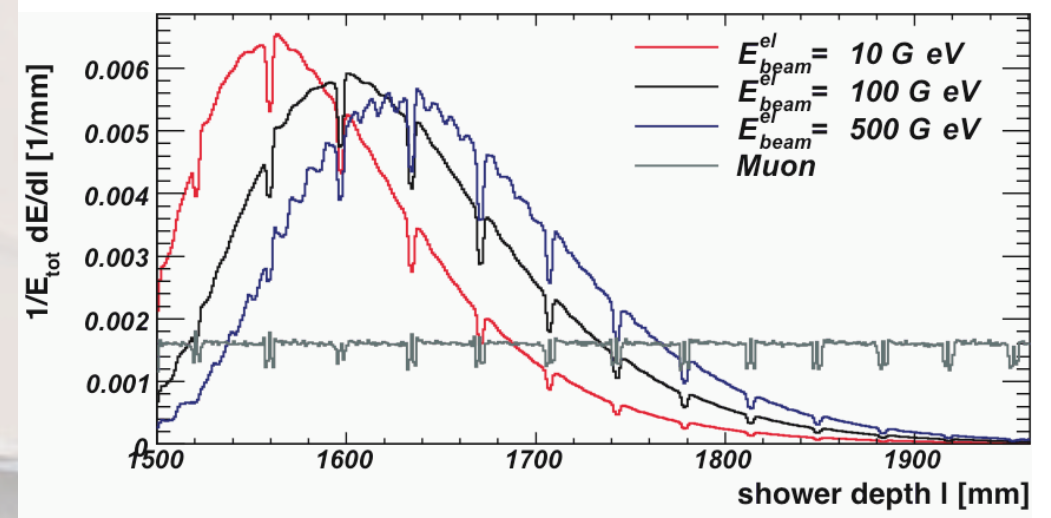

- Sampling Fraction (SF) not exactly constant!

- Depends on shower composition.

- Many short-ranged, low-energy particles are created and absorbed in the lead (much higher cross-

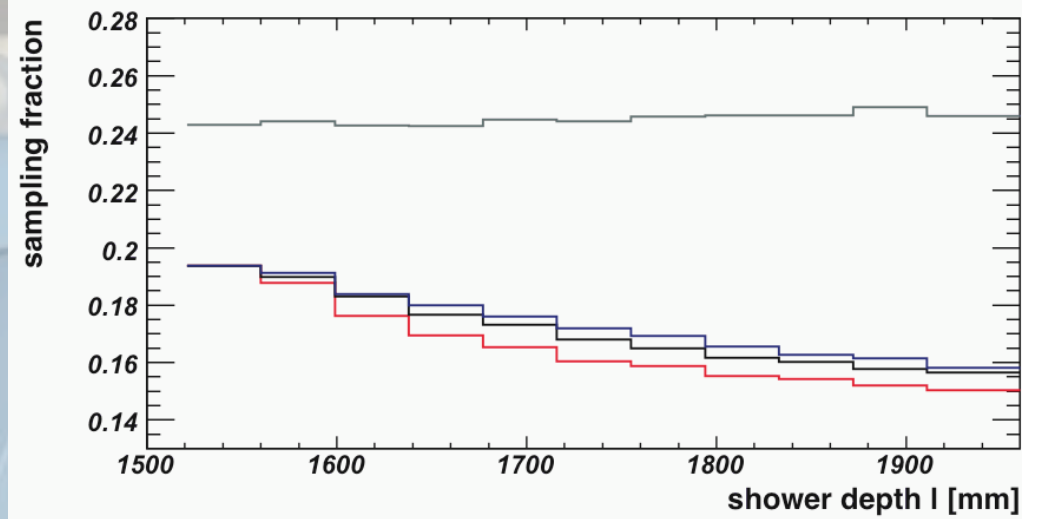
section for photo-electric effect than argon)

- Sampling Fraction decreases with depth and radius as such particles become more and more dominant.

- Use different SF for longitudinal compartments?

- Compromises resolution and linearity since shower depth fluctuates.

Use same sampling fraction for all compartments and apply energy-dependent correction factor 


\section{Final Calibration Formula}

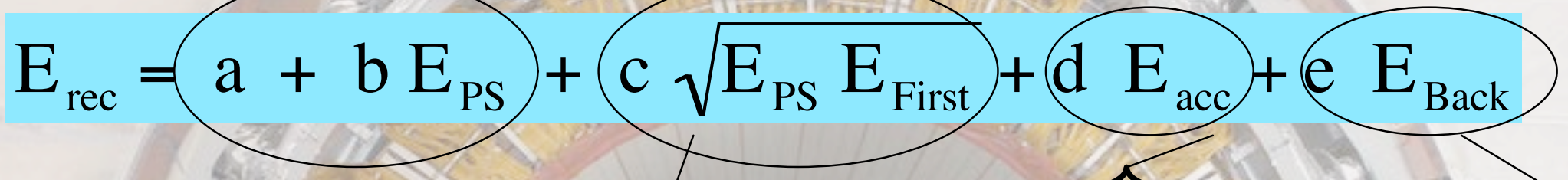

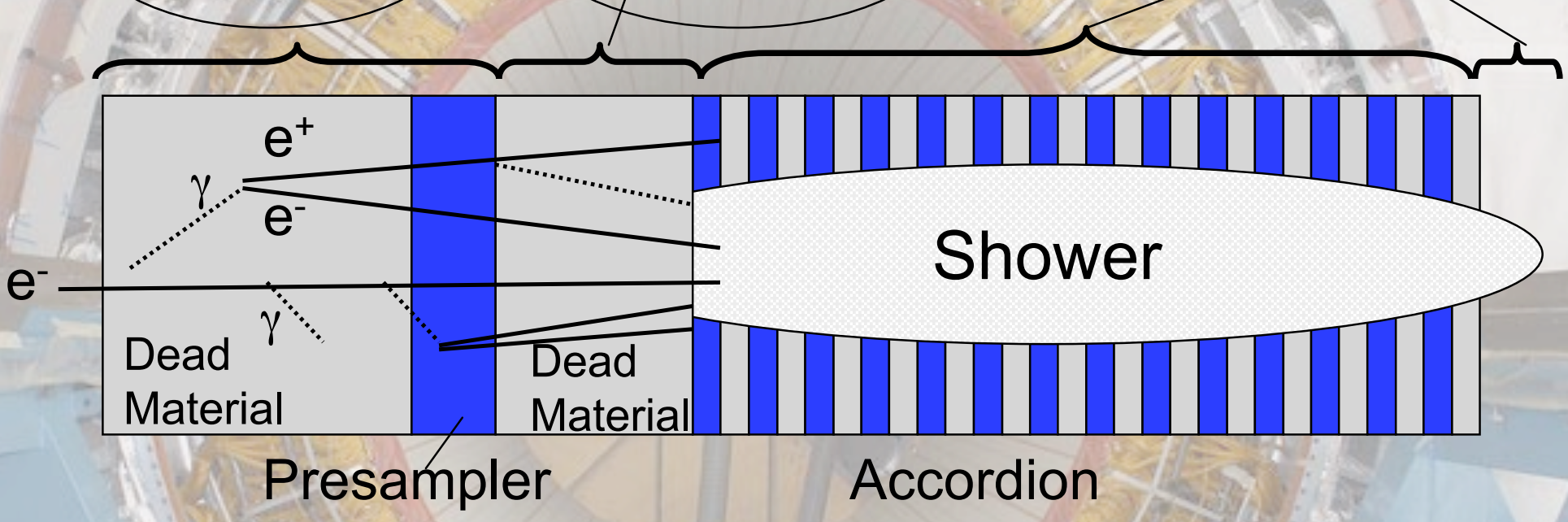

- Good linearity and resolution achieved

- Constants depend on impact point (upstream material) and on the energy.

- Can be parameterized.

- Constants are derived from a MC simulation of the detector setup. 


\section{Data Comparison (1)}

\section{- Most difficult issue:}

- Accurate description of upstream material

- Air and beam-pipe windows between energy-defining spectrometer and calorimeter $\left(\sim 0.15 \mathrm{X}_{0}\right)$

- Cables and electronics in the gap between Presampler and Accordion

- Plots shown use "equivalent material" in the geometry.

- Meanwhile better understood, new simulation of 2004 run being produced.

- More plots in M. Delmastro's talk
Comparison of energy fraction in each layer for $10 \mathrm{GeV}$ and $100 \mathrm{GeV}$ (2002-run)
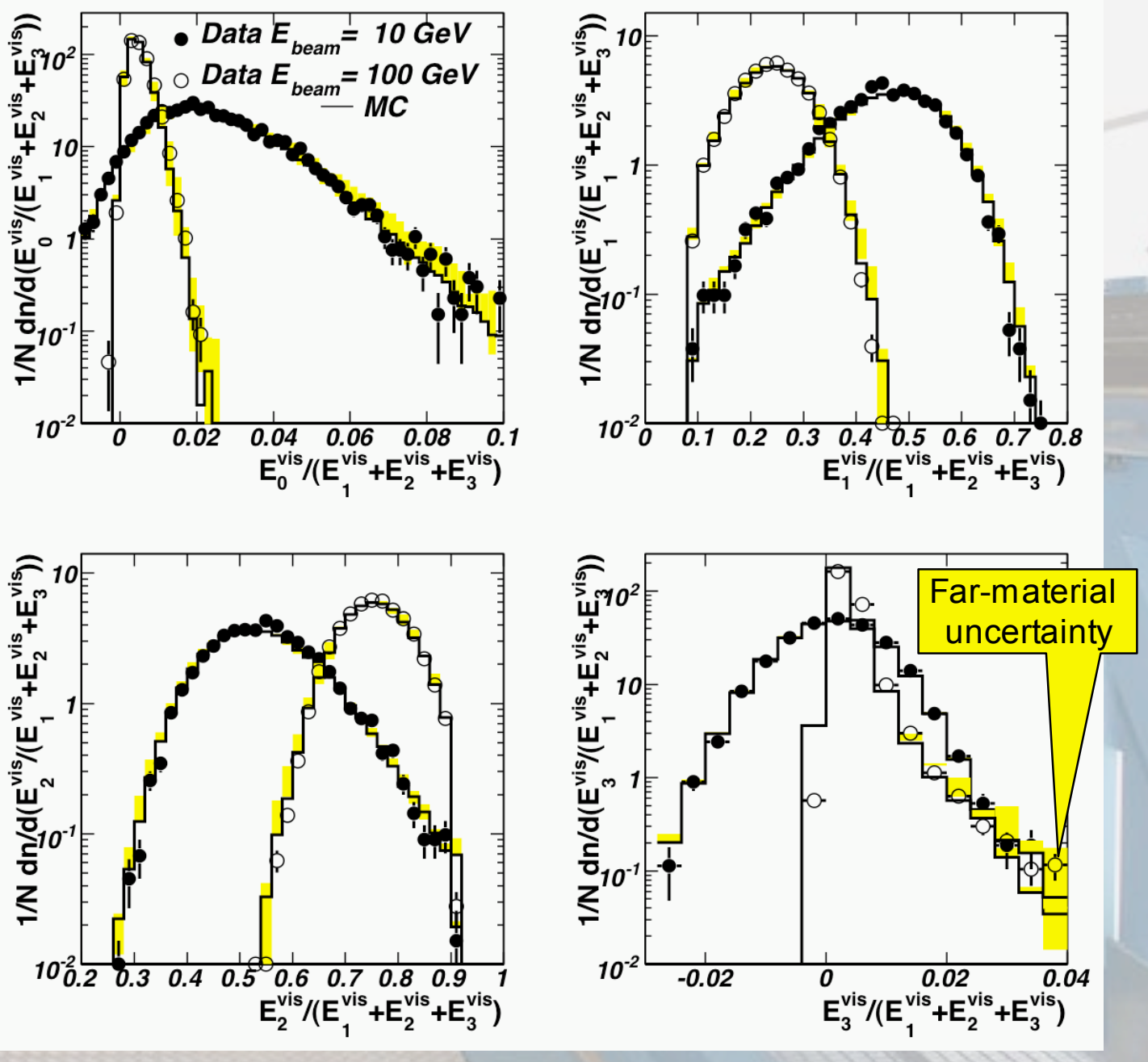


\section{MC-Data Comparison (2)}

Ratio of mean energy in each compartment for all energies and all material configuration (2004-run)
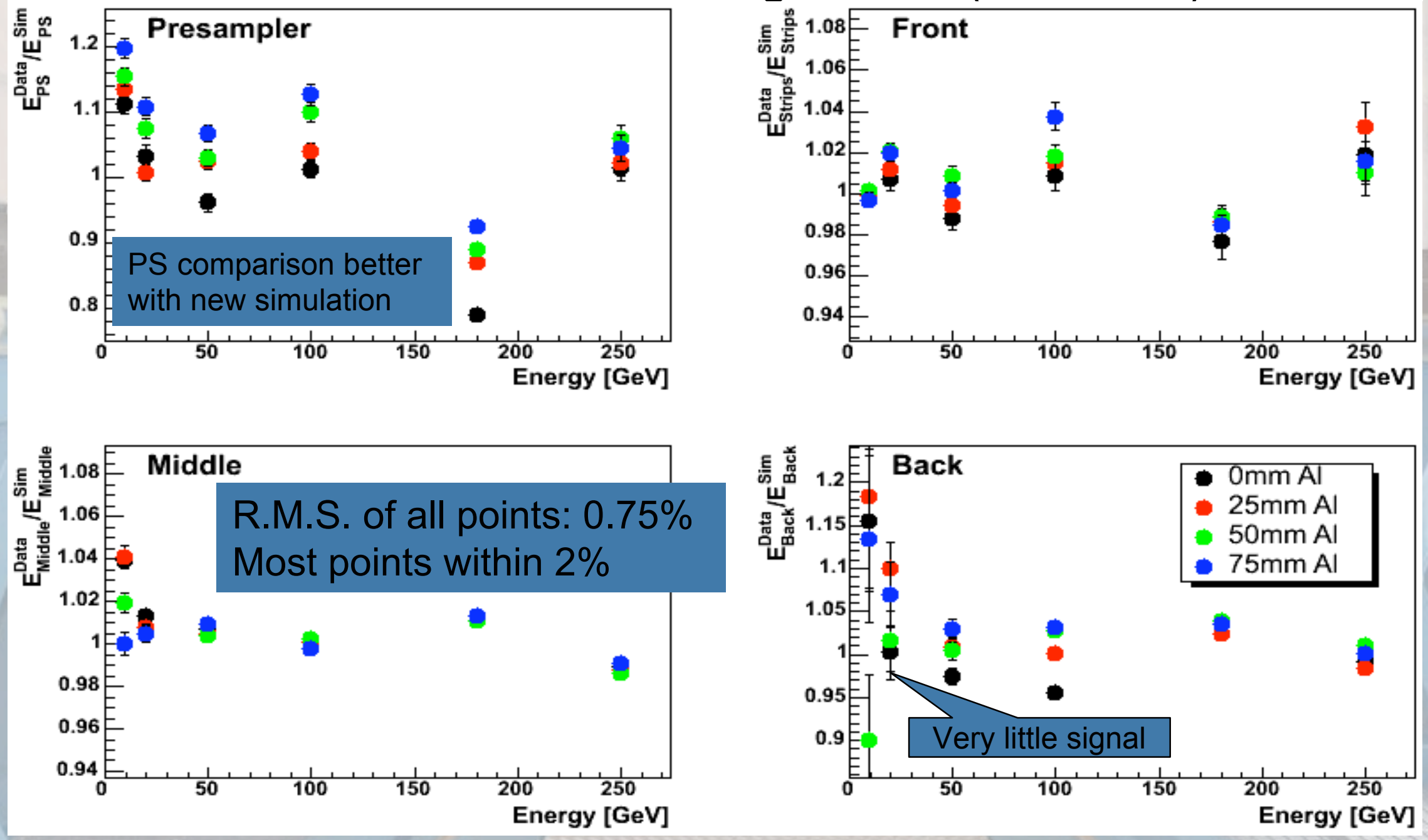


\section{Calibration Constants - 2002 Run}
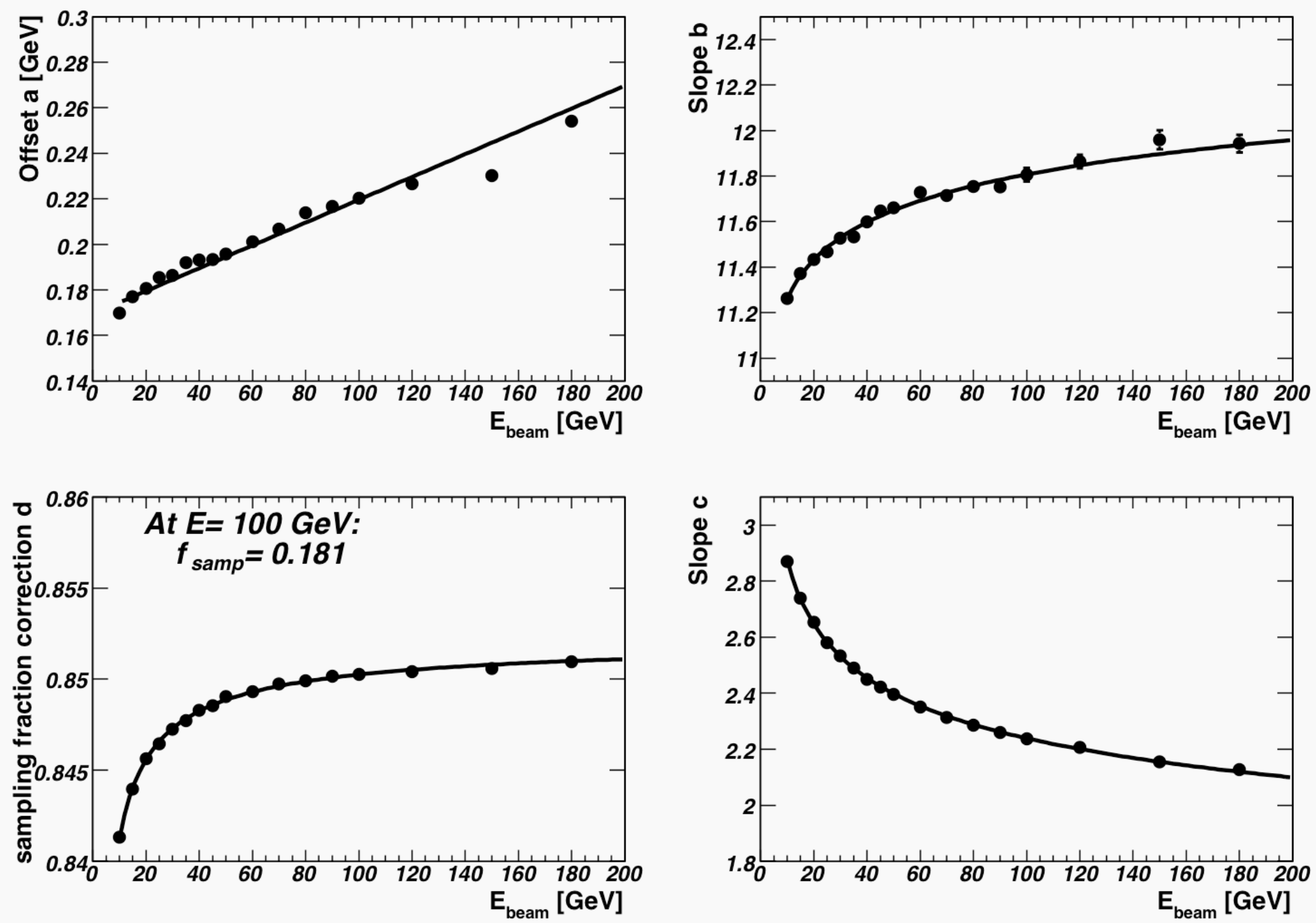


\section{Calibration Constants -2004 Run Dependence on upstream material}
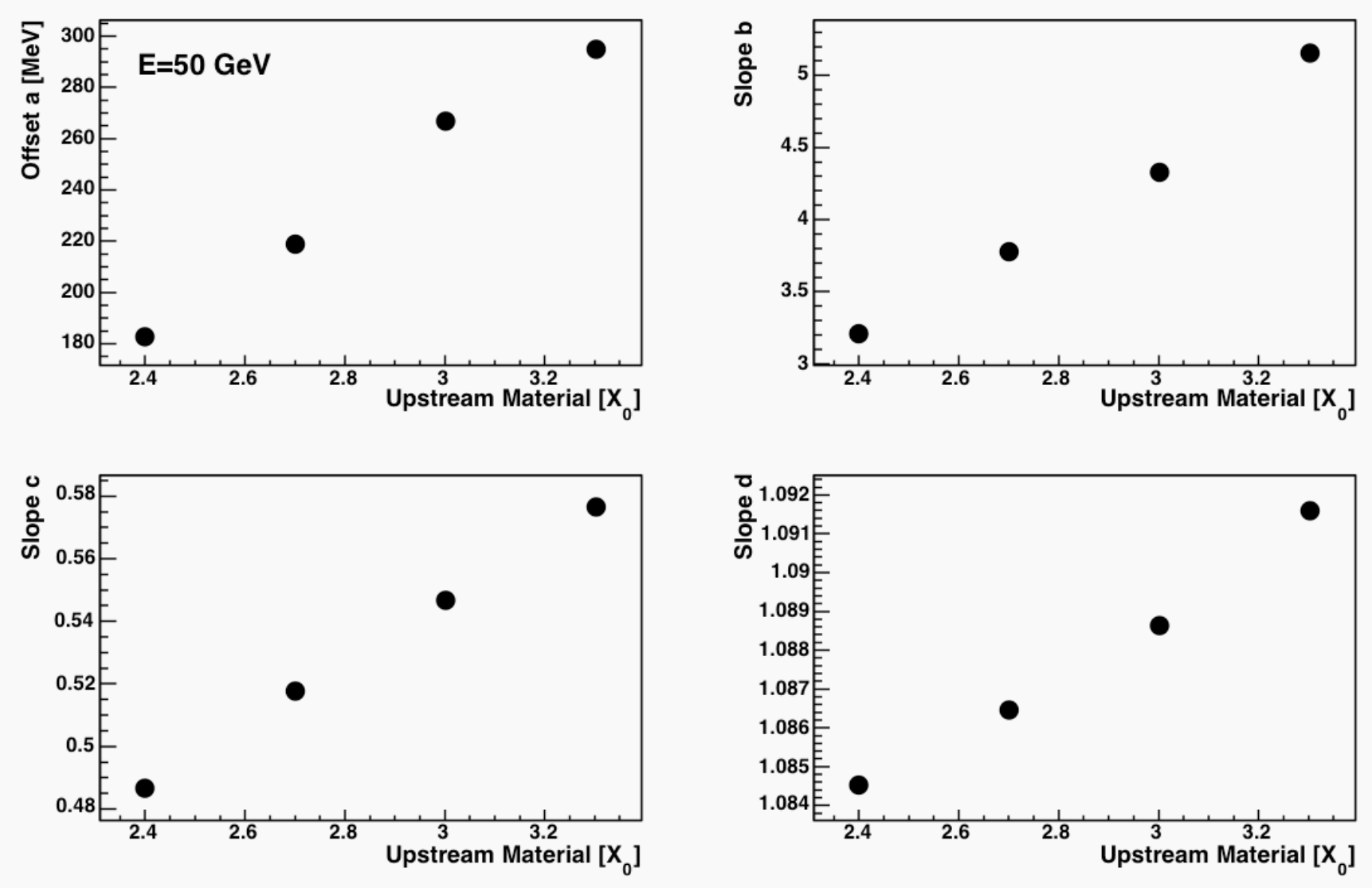

- All parameters rise when material is added

- More energy lost upstream, later part of the shower is measured. 


\section{Linearity and Resolution - 2002 Run}
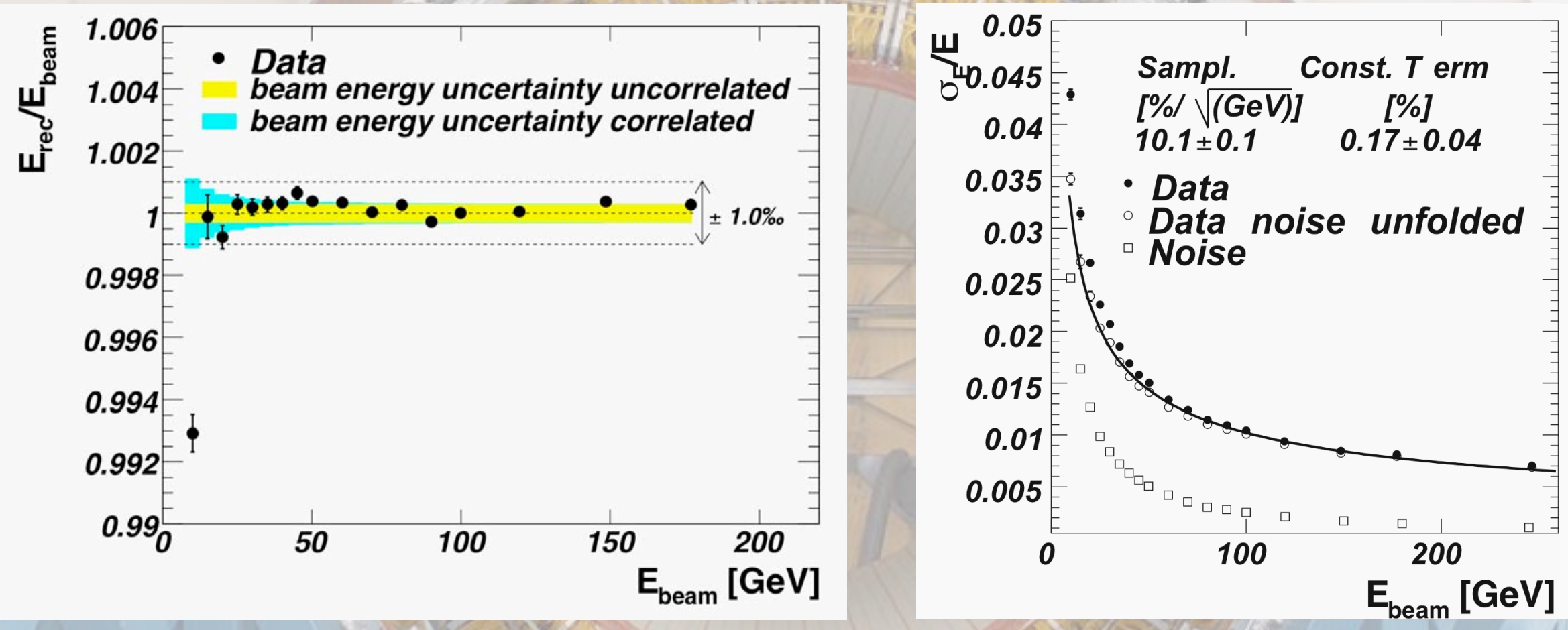

\section{- Procedure yields an excellent linearity (better than $\pm 0.1 \%$ for $\mathrm{E}>10 \mathrm{GeV}$ ) while preserving the resolution.}




\section{Linearity and Resolution-2004 Run}

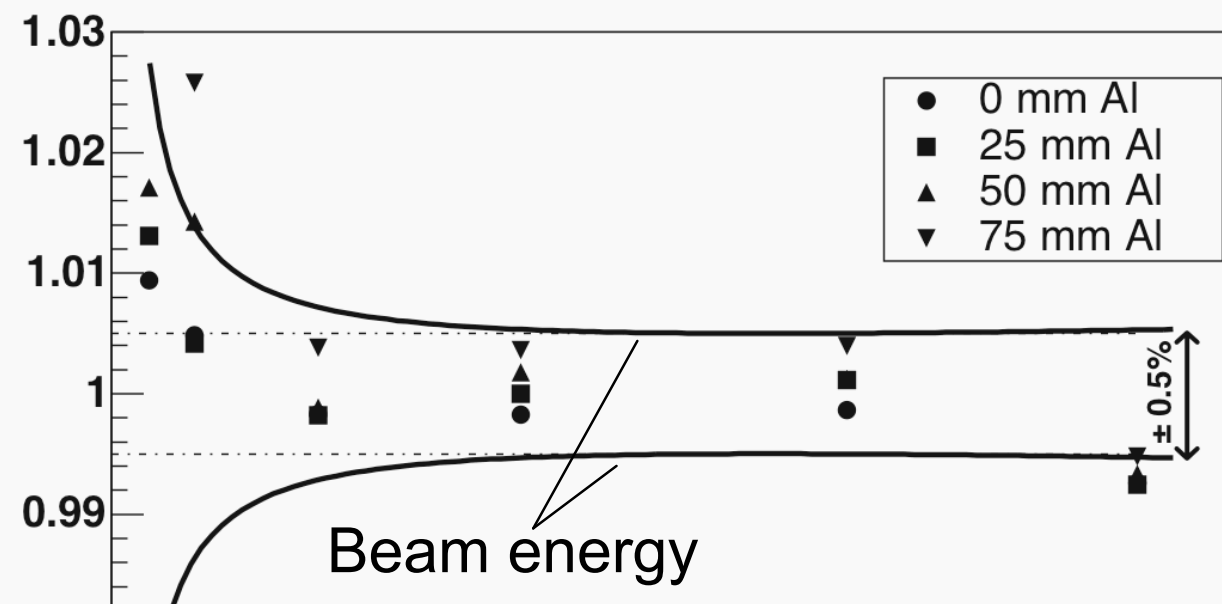

- Procedure works also for larger amounts of upstream matter

- Linear within the beam energy accuracy

- Work in progess...

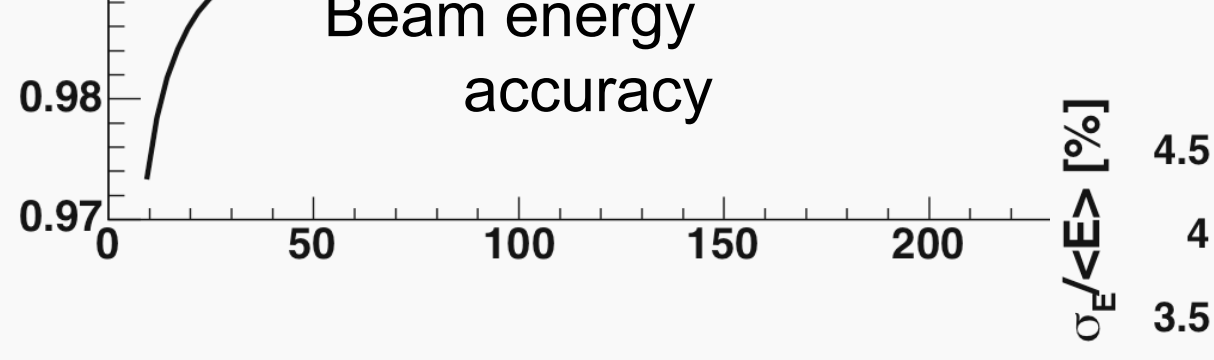

- $25 \mathrm{~mm} \mathrm{Al}$

- $50 \mathrm{~mm} \mathrm{~A}$

v $75 \mathrm{~mm} \mathrm{Al}$

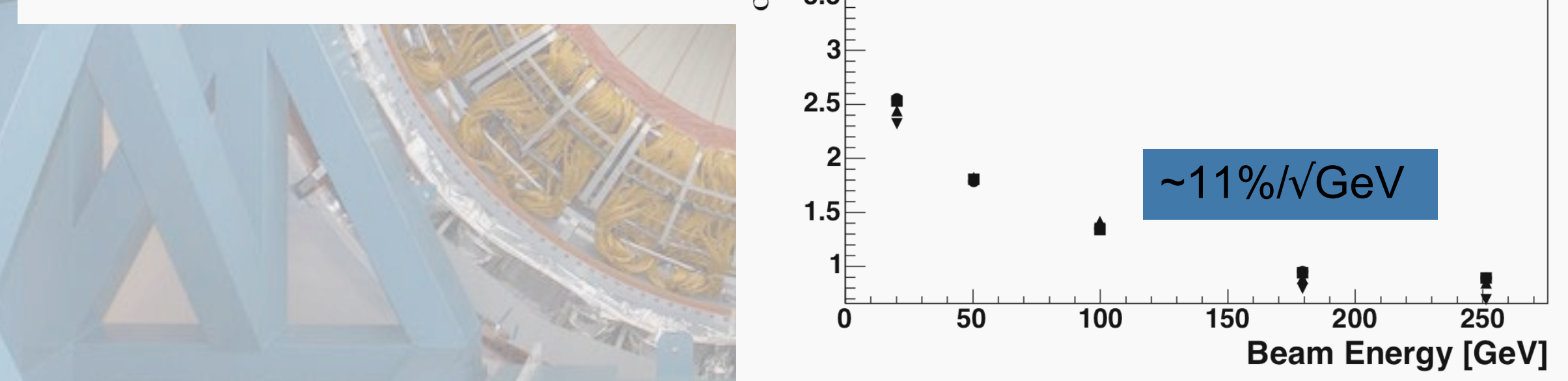




\section{Effect of wrongly estimated upstream material}

- Apply calibration constants derived for slightly different setup

- Upstream material overestimated by $0.3 X_{0}$

- Upstream material underestimated by $0.3 X_{0}$

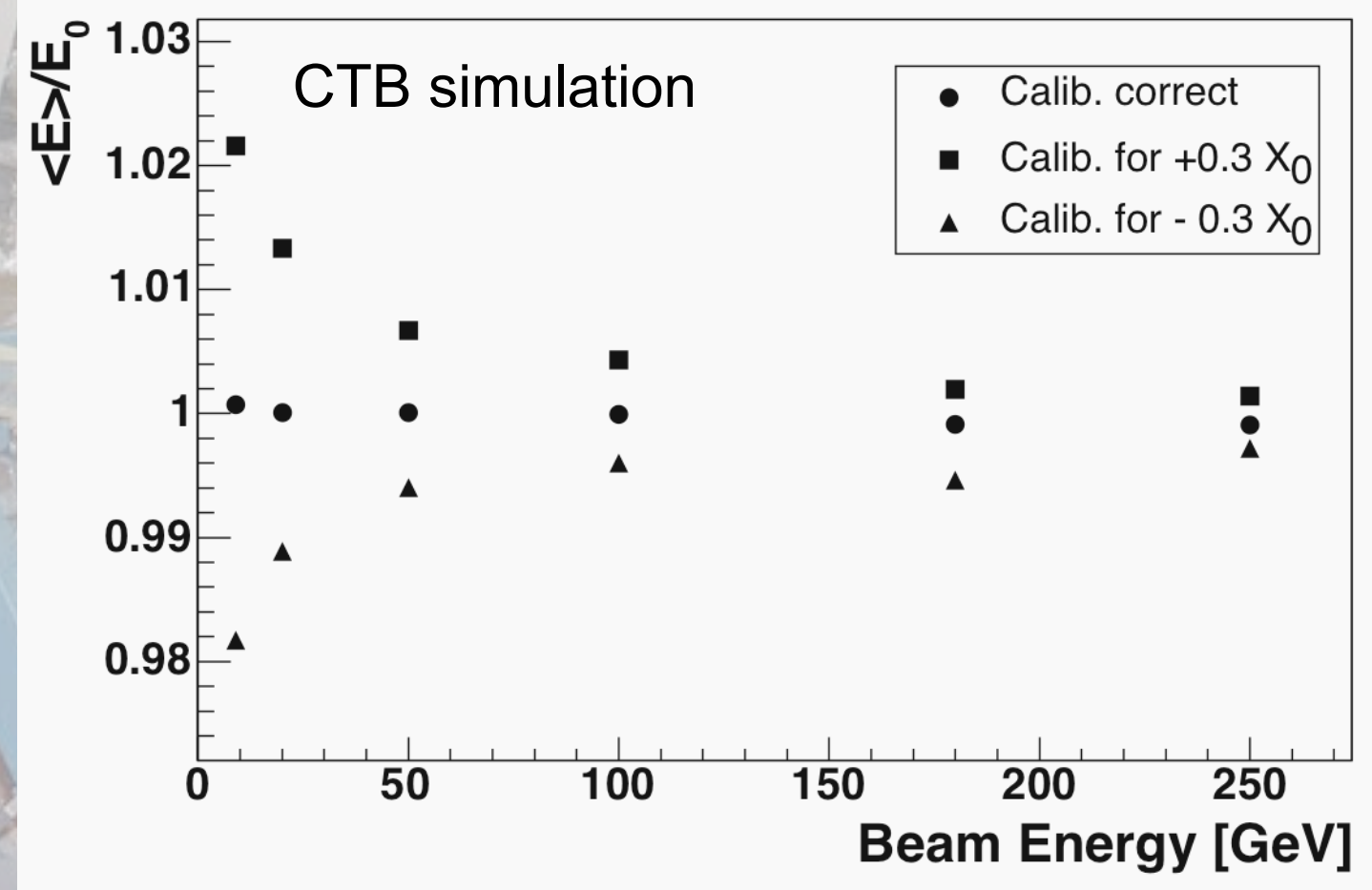

- Resulting error within $1 \%$ for energies at $50 \mathrm{GeV}$

- Initial material estimation in ATLAS won't be perfect ...... 


\section{Conclusions}

- Analysis of 2002 Linearity Scan almost finished.

- Linearity of $0.1 \%$ achieved

- Submitted to NIM for publication

- Analysis of 2004 Linearity/Material Scan well advanced.

- To be included in the analysis:

- More detailed simulation of upstream material distribution

- Better understanding of the beam energy accuracy

- Knowledge obtained from Testbeam analysis is incorporated in ATLAS software and will be important for proper energy reconstruction once data is coming. 